\title{
Association between serum adipsin and plaque vulnerability determined by optical coherence tomography in patients with coronary artery disease
}

\author{
Renhua Sun ${ }^{1,2}$, Yong Qiao ${ }^{1}$, Gaoliang Yan ${ }^{1}$, Dong Wang ${ }^{1}$, Wenjie Zuo ${ }^{1}$, Zhenjun Ji ${ }^{1}$, Xiaoguo Zhang ${ }^{1}$, \\ Yuyu Yao ${ }^{1}$, Genshan $\mathrm{Ma}^{1}$, Chengchun Tang ${ }^{1}$ \\ ${ }^{1}$ Department of Cardiology, Zhongda Hospital, School of Medicine, Southeast University, Nanjing, China; ${ }^{2}$ Department of Cardiology, Yancheng \\ No.1 People's Hospital, Yancheng, China \\ Contributions: (I) Conception and design: R Sun, C Tang; (II) Administrative support: Y Yao, G Ma, C Tang; (III) Provision of study materials or \\ patients: R Sun, Y Qiao, G Yan, D Wang, W Zuo, Z Ji; (IV) Collection and assembly of data: R Sun, X Zhang; (V) Data analysis and interpretation: \\ R Sun, W Zuo, Z Ji; (VI) Manuscript writing: All authors; (VII) Final approval of manuscript: All authors. \\ Correspondence to: Chengchun Tang, MD, PhD. Department of Cardiology, Zhongda Hospital, School of Medicine, Southeast University, No. 87 \\ Dingjiaqiao, Nanjing 210009, China. Email: tangchengchun@hotmail.com.
}

Background: Early identification of vulnerable plaques is important for patients with coronary artery disease (CAD) to reduce acute coronary events and improve their prognosis. We sought to examine the relationship between adipsin, an adipokine secreted from adipocytes, and plaque vulnerability in CAD patients.

Methods: A total of 103 plaques from 99 consecutive patients who underwent coronary angiography were assessed by optical coherence tomography. The serum level of adipsin was measured using enzyme-linked immunosorbent assay (ELISA). The accuracy of adipsin for detecting thin-cap fibroatheroma (TCFA) was determined by the area under the receiver operating characteristic curve (AUC).

Results: Of the 99 patients, 49 were classified into the low adipsin group and 50 into the high adipsin group according to the median level of serum adipsin $(2.43 \mu \mathrm{g} / \mathrm{mL})$. The plaques from the high adipsin group exhibited a greater lipid index $\left(2,700.0\right.$ vs. $\left.1,975.9^{\circ} \times \mathrm{mm}, \mathrm{P}=0.015\right)$ and an increased proportion of TCFAs (41.2\% vs. $21.2 \%, \mathrm{P}=0.028)$ compared with the low adipsin group. Serum adipsin was found to be negatively correlated with fibrous cap thickness $(\rho=-0.322, \mathrm{P}=0.002)$, while it was positively correlated with average lipid arc $(\rho=0.253, P=0.015)$, maximum lipid arc $(\rho=0.211, P=0.044)$, lipid core length $(\rho=0.241$, $\mathrm{P}=0.021)$, lipid index $(\rho=0.335, \mathrm{P}=0.001)$, and vulnerability score $(\rho=0.254, \mathrm{P}=0.014)$. Furthermore, adipsin had a significant association with TCFAs (OR: 1.290, 95\% CI: $1.048-1.589, \mathrm{P}=0.016$ ) in the multivariate analysis, while having a moderate diagnostic accuracy for TCFAs (AUC: 0.710, 95\% CI: 0.602-0.817, $\mathrm{P}<0.001)$.

Conclusions: Our findings suggest that serum adipsin is significantly and positively correlated with the incidence of TCFAs. The application of adipsin as a biomarker may offer improvement in the diagnosis of vulnerable plaques and clinical benefits for CAD patients.

Keywords: Adipsin; plaque vulnerability; thin-cap fibroatheroma (TCFA); coronary artery disease (CAD); optical coherence tomography

Submitted Jan 21, 2021. Accepted for publication Mar 29, 2021.

doi: $10.21037 /$ jtd-21-259

View this article at: http://dx.doi.org/10.21037/jtd-21-259 


\section{Introduction}

Although great progress has been made in our understanding of atherosclerosis and cardiovascular diseases over the past decades, their related complications are a serious problem for society due to high morbidity and mortality (1). Sudden rupture of coronary atherosclerotic plaques often causes thrombus formation and subsequent acute myocardial infarction (AMI) in patients with pre-existing coronary artery disease (CAD). This pathophysiological process can lead to rapid deterioration of patients' condition and poor prognosis or even death. Thus, the concept of vulnerable plaques, defined as high-risk, unstable plaques prone to cause acute coronary syndrome, was introduced to predict the occurrence of adverse cardiovascular events and achieve early risk stratification of CAD patients $(2,3)$. Thin-cap fibroatheroma (TCFA), characterized as a lipid-rich plaque with a thin fibrous cap $(\leq 65 \mu \mathrm{m})$, is a hallmark of plaque vulnerability (4). Inflamed TCFA is the most common form of vulnerable plaques and responsible for $60-70 \%$ of acute coronary events (5). Discovering new biomarkers of TCFA is of great significance for improving patient management and reducing residual risk.

Robust evidence has shown that atherosclerosis is a chronic, sterile inflammatory disease in which various elements of the immune system participate (6). The complement system may play a pivotal role in this pathological process as a crucial link between adaptive and innate immunity, while its alternative pathway is involved in a variety of human diseases (7). Adipsin, first described as an adipokine (8) and later identified as complement component factor D (9), catalyzes the rate-limiting step of the alternative pathway to regulate complement activation (10). It is mainly secreted by adipose tissue, macrophages, and monocytes (9). Moreover, adipsin is associated with various pathophysiological characteristics underlying atherosclerosis, including low-grade inflammation, endothelial dysfunction, ischemia-reperfusion, and lipid metabolism (11-13). Recently, it has been demonstrated that higher serum adipsin can predict not only rehospitalization and all-cause death in patients with $\mathrm{CAD}$, but also their future incidence of acute coronary events (14). More importantly, an increased level of adipsin in unstable plaques was detected by immunostaining among AMI patients (14). It seems that adipsin may be related to the destabilization of atherosclerotic plaques.

As a high-resolution intravascular imaging modality, optical coherence tomography (OCT) was recently introduced to accurately assess coronary plaque microstructure and to identify vulnerable plaque characteristics (15-17). However, there has been no previous study using this sensitive intracoronary approach to examine the association between serum adipsin and vulnerable plaque characteristics. The present study aimed to investigate the correlation between serum adipsin and OCT-determined vulnerable plaques in patients with $\mathrm{CAD}$, and evaluate the possibility of adipsin as a potential biomarker for vulnerable plaques. We present the following article in accordance with the STROBE reporting checklist (available at http://dx.doi.org/10.21037/jtd-21-259).

\section{Methods}

\section{Subjects and study design}

The study prospectively enrolled 106 consecutive CAD patients who underwent both coronary angiography and simultaneous OCT examination from April 2018 to July 2020 at Zhongda Hospital, Southeast University. The inclusion criteria were (I) age $\geq 18$ years and (II) interrogated lesions with visual percent diameter stenosis (\%DS) $\geq 30 \%$. Patients or lesions were excluded if they met one of the following criteria: (I) previous history of coronary artery bypass graft; (II) extreme tortuosity, in-stent restenosis, leftmain stenosis, heavy calcification, or chronic total occlusion; (III) renal dysfunction with an estimated glomerular filtration rate (eGFR) $<60 \mathrm{~mL} / \mathrm{min}$; (IV) congestive heart failure with left ventricular ejection fraction (LVEF) $<40 \%$; and (V) other inflammatory conditions, including cancer, infection, and autoimmune diseases. After screening 106 patients, 7 patients were excluded due to in-stent restenosis $(n=6)$ and poor image quality $(n=1)$, resulting in a total of 99 patients with 103 lesions eligible for final analysis. Following the Declaration of Helsinki (as revised in 2013), this study was approved by the institutional ethics committee of Zhongda Hospital, School of Medicine, Southeast University, and all patients provided written informed consent.

\section{Coronary angiography and OCT analysis}

All included patients received coronary angiography using a standard method via a transradial approach. Two and 4 angiographic projections were routinely acquired for the right and left coronary arteries, respectively. All angiograms were independently reviewed, and $\%$ DS of interrogated lesions was visually assessed by experienced interventionalists who were blinded to clinical characteristics, OCT results, and serum adipsin. The frequency-domain OCT C7XR system (St. Jude Medical, 

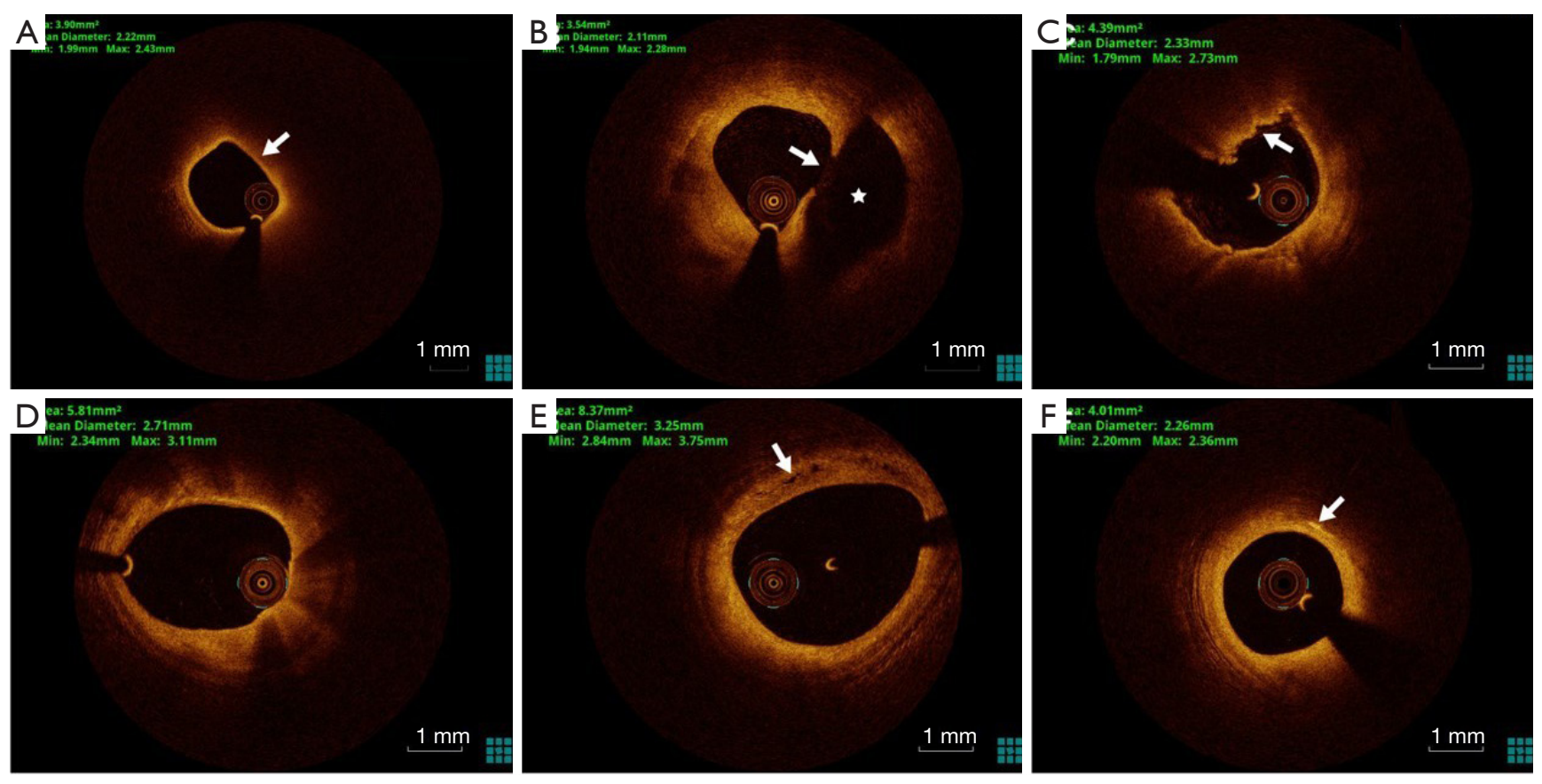

Figure 1 Representative OCT images. (A) A thin-cap fibroatheroma (TCFA) with the thinnest fibrous cap $<65 \mu \mathrm{m}$ (the white arrow) and with lipid arc $>180^{\circ}$; (B) plaque rupture: presence of discontinued fibrous cap (the white arrow) and cavity formation (the white asterisk); (C) plaque erosion: residual white thrombus (the white arrow) underlying an intact fibrous cap; (D) macrophage accumulation: signal-rich, distinct, or confluent punctuate regions that had a higher intensity than background speckle noise; (E) microchannel: a black hole (the white arrow) with diameter of 50-300 $\mu \mathrm{m}$ that neither reflects the signal nor connects to the lumen, which was present in at least 3 consecutive frames; (F) cholesterol crystal: a thin, linear, and highly-backscattering region (the white arrow) within the plaque.

Westford, MA, USA) was used to acquire OCT images. In brief, a 2.7-French OCT catheter was positioned distal to the lesion after intracoronary infusion of $100-200 \mu \mathrm{g}$ nitroglycerin. Then, the catheter was automatically pulled back to the proximal segment at a speed of $25 \mathrm{~mm} / \mathrm{s}$ (100 frames/s) during blood clearance that was achieved by injecting iso-osmotic contrast medium. For off-line measurements ( $1.0 \mathrm{~mm}$ interval scale), all OCT images were analyzed with a dedicated workstation by experienced technicians who were blinded to the clinical data.

Plaque characteristics were determined according to standard definitions (17). Based on OCT images, plaques were divided into 2 categories: (I) lipid plaque (a poor signal region with a diffuse border) and (II) fibrous plaque (a homogeneous region with high backscattering) (17). For lipid plaques, lipid length was measured in the longitudinal direction, and the lipid arc was measured through the entire length of each plaque. The lipid index was calculated by multiplying lipid length and average lipid arc (18). The fibrous cap was defined as a signal-rich homogenous layer overlying the lipid plaque. Moreover, fibrous cap thickness (FCT) was measured 3 times at the thinnest part of the fibrous cap, and its average value was calculated. A thincap fibroatheroma (TCFA) was characterized as a lipidrich plaque with a maximal lipid arc $>90^{\circ}$ and thinnest FCT $\leq 65 \mu \mathrm{m}$ (Figure 1A) (4). Plaque rupture was defined as discontinued fibrous cap and cavity formation within the plaque (Figure 1B) (19). Plaque erosion was defined by the presence of an attached thrombus overlying an intact plaque without fibrous cap disruption (Figure 1C) (20). Macrophage accumulation was identified as signal-rich, distinct, or confluent punctuate regions that had a higher intensity than background speckle noise (Figure 1D) (17). The microchannel was defined as a black hole that neither reflected the signal nor connected to the lumen and was present in at least 3 consecutive frames (Figure 1E) $(17,21)$. Cholesterol crystals were characterized as thin, linear, and highly backscattering regions within the plaque (Figure $1 F$ ) $(17,22)$. The vulnerability score was based on the presence of vulnerability markers on OCT images, such as TCFAs, lipid 
pools, microchannels, and macrophage accumulation (23). This value was calculated as the total number of all these vulnerability markers in 100 consecutive OCT frames (25 mm length) (24).

\section{Adipsin measurement}

Blood samples were collected via radial access before heparinization using vacutainer tubes. These samples were maintained at $4{ }^{\circ} \mathrm{C}$ and processed within 2 hours before centrifugation at 3,000 rpm for 10 minutes. Serum samples were separated and then stored at $-80{ }^{\circ} \mathrm{C}$ until further analysis. Serum adipsin was determined using a dedicated enzyme-linked immunosorbent assay (ELISA) kit (Catalog Number: CSB-E14369h, Wuhan, China) according to the manufacturer's instructions. All ELISA data were analyzed in relation to the standard curve. Each sample was measured twice to acquire the mean value. The ELISA plates were measured using a BioTek ELx800 microplate reader (BioTek Instruments, Winooski, Vermont, USA) and analyzed with supporting software (KC Junior).

\section{Statistical analysis}

Normality was examined using histograms and Q-Q plots. Continuous variables are presented as the mean \pm standard deviation (SD) or median $\left(25^{\text {th }}-75^{\text {th }}\right.$ percentiles) as appropriate. Student's t or Mann-Whitney U tests were used to detect the differences between the 2 groups. Categorical variables are presented as counts (percentages) and were compared with a chi-square test. Spearman correlation analysis was used to assess the association between serum adipsin and OCT parameters. To examine whether serum adipsin was independently associated with TCFAs, we used logistic regression analysis to exclude confounding factors. The receiver operating characteristic (ROC) curve was generated for adipsin to assess its utility for detecting TCFAs, and its optimal cut-off point was determined by the Youden index. All data were analyzed by SPSS version 25.0 (IBM Inc., Chicago, Illinois, USA) and MedCalc version 19.0.7 (MedCalc Software bvba, Ostend, Belgium). A twotailed $\mathrm{P}$ value $<0.05$ was considered statistically significant.

\section{Results}

\section{Baseline patient and lesion characteristics}

A total of 99 patients with 103 interrogated lesions were included in the present study. All patients were divided into a low-level group $(n=49)$ or a high-level group $(n=50)$ according to the median value of serum adipsin $(2.43 \mu \mathrm{g} / \mathrm{mL})$. Baseline patient characteristics and laboratory tests are detailed in Table 1. No significant differences were observed between the 2 groups in age, sex, risk factors, clinical presentations, or medications. There was a higher level of serum creatinine in the high adipsin group than in the low adipsin group $(\mathrm{P}=0.007)$. The adipsin level was 1.89 (1.64-2.17) $\mu \mathrm{g} / \mathrm{mL}$ in the low group and $4.45(3.52-7.12)$ $\mu \mathrm{g} / \mathrm{mL}$ in the high group $(\mathrm{P}<0.001)$. The other laboratory parameters were not significantly different between the 2 groups.

Angiographic and OCT findings were compared between the 2 groups and are shown in Table 2. There was no difference in the location of plaques between the 2 groups. Visual \%DS in the high adipsin group was greater than that in the low adipsin group (45\% vs. $40 \%, \mathrm{P}=0.006)$. A total of 92 lipid-rich plaques $(89.3 \%)$ were detected by OCT, and 32 TCFAs $(31.1 \%)$ were found among these plaques. Lipid-rich plaques in the low adipsin group had a smaller lipid index than those in the high adipsin group (1,975.9 vs. $\left.2,700.0^{\circ} \times \mathrm{mm}, \mathrm{P}=0.015\right)$. Compared with the low adipsin group, lesions from the high adipsin group had a greater proportion of TCFAs (41.2\% vs. $21.2 \%, \mathrm{P}=0.028)$.

\section{Correlation between serum adipsin and OCT parameters}

Figure 2 shows the correlation between serum adipsin and OCT parameters. Serum adipsin was negatively correlated with FCT $(\rho=-0.322, \mathrm{P}=0.002)$, while it was also positively correlated with average lipid arc $(\rho=0.253, \mathrm{P}=0.015)$, maximum lipid arc $(\rho=0.211, P=0.044)$, lipid core length $(\rho=0.241, P=0.021)$, lipid index $(\rho=0.335, P=0.001)$, and vulnerability score $(\rho=0.254, P=0.014)$.

\section{Adipsin as an independent factor predicting OCT-detected TCFAs}

To further confirm the association between serum adipsin and plaque vulnerability, we performed logistic regression analysis to identify independent predictive factors of TCFAs (Table 3). The results at the univariate level demonstrated that male sex (OR: 2.097, 95\% CI: 0.851-5.164, P = 0.107), triglycerides (OR: 1.290, 95\% CI: 0.948-1.756, $\mathrm{P}=0.106$ ), total cholesterol (OR: 1.463, 95\% CI: 0.997-2.148, $\mathrm{P}=0.052$ ), low-density lipoprotein cholesterol (OR: 1.921, 95\% CI: 1.059-3.486, P=0.032), adipsin (OR: 1.330, 95\% CI: $1.114-1.588, \mathrm{P}=0.002)$, acute coronary syndrome (OR: 
Table 1 Baseline characteristics of included patients

\begin{tabular}{|c|c|c|c|c|}
\hline \multirow{2}{*}{ Variables } & \multirow{2}{*}{ Total patients $(\mathrm{n}=99)$} & \multicolumn{2}{|c|}{ Adipsin level } & \multirow{2}{*}{$P$ value } \\
\hline & & Low $(n=49)$ & High $(n=50)$ & \\
\hline Age, years & $60.6 \pm 12.0$ & $60.0 \pm 9.4$ & $61.2 \pm 14.2$ & 0.617 \\
\hline $\mathrm{BMI}, \mathrm{kg} / \mathrm{m}^{2}$ & $25.0 \pm 3.57$ & $25.18 \pm 3.65$ & $24.92 \pm 3.52$ & 0.722 \\
\hline LVEF, \% & $68.10 \pm 8.95$ & $67.43 \pm 7.79$ & $68.77 \pm 10.00$ & 0.460 \\
\hline Prior $\mathrm{PCl}$ & $9(9.1)$ & $3(6.1)$ & $6(12.0)$ & 0.487 \\
\hline \multicolumn{5}{|l|}{ Risk factors } \\
\hline Hypertension & $69(69.7)$ & $34(69.4)$ & $35(70.0)$ & 0.947 \\
\hline Diabetes mellitus & $20(20.2)$ & $9(18.4)$ & $11(22.0)$ & 0.653 \\
\hline STEMI & $8(8.1)$ & $4(8.2)$ & $4(8.0)$ & 1.000 \\
\hline NSTEMI & $26(26.3)$ & $13(26.5)$ & $13(26.0)$ & 0.952 \\
\hline Unstable angina & $32(32.3)$ & $19(38.8)$ & $13(26.0)$ & 0.174 \\
\hline Stable angina & $33(33.3)$ & $13(26.5)$ & $20(40.0)$ & 0.155 \\
\hline \multicolumn{5}{|l|}{ Medications } \\
\hline Aspirin & $80(80.8)$ & $38(77.6)$ & $42(84.0)$ & 0.415 \\
\hline P2Y12 receptor antagonists & $65(65.7)$ & $32(65.3)$ & $33(66.0)$ & 0.942 \\
\hline$\beta$-blockers & $66(66.7)$ & $33(67.3)$ & $33(66.0)$ & 0.887 \\
\hline Serum creatinine, $\mu \mathrm{mol} / \mathrm{L}$ & $69(58-80)$ & $65(55-73)$ & $74(59-92)$ & 0.007 \\
\hline Uric acid, $\mu \mathrm{mol} / \mathrm{L}$ & $338.84 \pm 102.92$ & $331.71 \pm 114.99$ & $345.82 \pm 90.16$ & 0.498 \\
\hline $\mathrm{TC}, \mathrm{mmol} / \mathrm{L}$ & $4.43 \pm 1.06$ & $4.34 \pm 1.02$ & $4.53 \pm 1.11$ & 0.370 \\
\hline $\mathrm{TG}, \mathrm{mmol} / \mathrm{L}$ & $1.45(1.08-2.15)$ & $1.39(0.97-2.18)$ & $1.48(1.14-2.14)$ & 0.443 \\
\hline LDL-C, mmol/L & $2.58 \pm 0.74$ & $2.52 \pm 0.74$ & $2.63 \pm 0.74$ & 0.458 \\
\hline HDL-C, mmol/L & $1.18 \pm 0.28$ & $1.15 \pm 0.27$ & $1.20 \pm 0.29$ & 0.394 \\
\hline ApoA1, g/L & $1.22 \pm 0.27$ & $1.23 \pm 0.31$ & $1.21 \pm 0.24$ & 0.811 \\
\hline ApoB, g/L & $0.77 \pm 0.19$ & $0.78 \pm 0.18$ & $0.76 \pm 0.20$ & 0.554 \\
\hline $\mathrm{Lp}(\mathrm{a}), \mathrm{mg} / \mathrm{L}$ & $125(53-358)$ & $143(48-398)$ & $117(64.25-317.75)$ & 0.763 \\
\hline Adipsin, $\mu \mathrm{g} / \mathrm{ml}$ & $2.43(1.89-4.48)$ & $1.89(1.64-2.17)$ & $4.45(3.52-7.12)$ & $<0.001$ \\
\hline
\end{tabular}

Values are presented as mean \pm standard deviation, median $\left(25^{\text {th }}-75^{\text {th }}\right.$ percentile), or $n(\%)$. ACEl, angiotensin-converting-enzyme inhibitor; ARB, angiotensin receptor blocker; BMI, body mass index; CCBs, calcium channel blockers; FBG, fasting blood glucose; HDL-C, highdensity lipoprotein cholesterol; LDL-C, low-density lipoprotein cholesterol; LVEF, left ventricular ejection fraction; MI, myocardial infarction; NSTEMI, non-ST-segment elevation myocardial infarction; PCI, percutaneous coronary intervention; STEMI, ST-segment elevation myocardial infarction; TC, total cholesterol; TG, triglycerides. 
Table 2 Angiographic and OCT findings of included lesions

\begin{tabular}{|c|c|c|c|c|}
\hline Variables & Total lesions $(n=103)$ & \multicolumn{2}{|c|}{ Adipsin level } & $P$ value \\
\hline \multicolumn{5}{|l|}{ Angiographic findings } \\
\hline Location & & & & 0.815 \\
\hline LAD & $73(70.9)$ & $38(73.1)$ & $35(68.6)$ & \\
\hline RCA & $20(19.4)$ & $10(19.2)$ & $10(19.6)$ & \\
\hline Visual \%DS & $45(40-50)$ & $40(30-45)$ & $45(40-55)$ & 0.006 \\
\hline \multicolumn{5}{|l|}{ OCT findings } \\
\hline Lipid-rich plaque & $92(89.3)$ & $44(84.6)$ & $48(94.1)$ & 0.118 \\
\hline Average lipid arc, ${ }^{\circ}$ & $160.1 \pm 52.9$ & $150.3 \pm 51.9$ & $170.0 \pm 52.6$ & 0.091 \\
\hline Maximum lipid arc, ${ }^{\circ}$ & $270(180-360)$ & $260(180-353)$ & $290(185-360)$ & 0.316 \\
\hline Lipid index, ${ }^{\circ} \times \mathrm{mm}$ & $2,375.7(1,285.0-3,565.3)$ & $1,975.9(834.5-2,958.6)$ & $2,700.0(1,673.7-3,863.3)$ & 0.015 \\
\hline Fibrous plaque & $11(10.7)$ & $8(15.4)$ & $3(5.9)$ & 0.118 \\
\hline Calcification & $47(45.6)$ & $21(40.4)$ & $26(51.0)$ & 0.280 \\
\hline Macrophage accumulation & $40(38.8)$ & $21(40.4)$ & $19(37.3)$ & 0.745 \\
\hline Microchannel & $39(37.9)$ & $19(36.5)$ & $20(39.2)$ & 0.779 \\
\hline Cholesterol crystal & $33(32.0)$ & $17(32.7)$ & $16(31.4)$ & 0.886 \\
\hline
\end{tabular}

Values are presented as mean \pm standard deviation, median $\left(25^{\text {th }}-75^{\text {th }}\right.$ percentile), or $n$ (\%). \%DS, percent diameter stenosis; FCT, fibrous cap thickness; LAD, left anterior descending artery; LCX, left circumflex artery; OCT, optical coherence tomography; RCA, right coronary artery; TCFA, thin-cap fibroatheroma.

5.122, 95\% CI: $1.624-16.154, \mathrm{P}=0.005)$, and visual \% DS (OR: $1.084,95 \%$ CI: $1.045-1.125$, P <0.001) were possible predictors. After including them in a multivariate model, only adipsin (adjusted OR: 1.290, 95\% CI: 1.048-1.589, $\mathrm{P}=0.016$ ), visual \%DS (adjusted OR: 1.086, 95\% CI: $1.037-$ $1.137, \mathrm{P}<0.001$ ), and acute coronary syndrome (adjusted OR: 4.058 , 95\% CI: $1.016-16.199, \mathrm{P}=0.047$ ) remained statistically significant, suggesting that adipsin was an independent factor predicting the presence of TCFA. As presented in Figure 3A, patients with TCFAs had a higher level of serum adipsin than those without TCFAs (3.82 vs. $2.20 \mu \mathrm{g} / \mathrm{mL}, \mathrm{P}<0.001)$. The ROC analysis showed that serum adipsin could partially predict TCFA (area under the ROC curve (AUC): 0.710, 95\% CI: 0.602-0.817, $\mathrm{P}<0.001$ ), with an optimal cut-off of $3.50 \mu \mathrm{g} / \mathrm{mL}$ (sensitivity $65.6 \%$, specificity $74.7 \%$ ) (Figure $3 B$ ).

\section{Discussion}

This is the first study investigating the correlation between serum adipsin and OCT-detected plaque vulnerability in CAD patients. Our main findings were as follows: (I) serum 

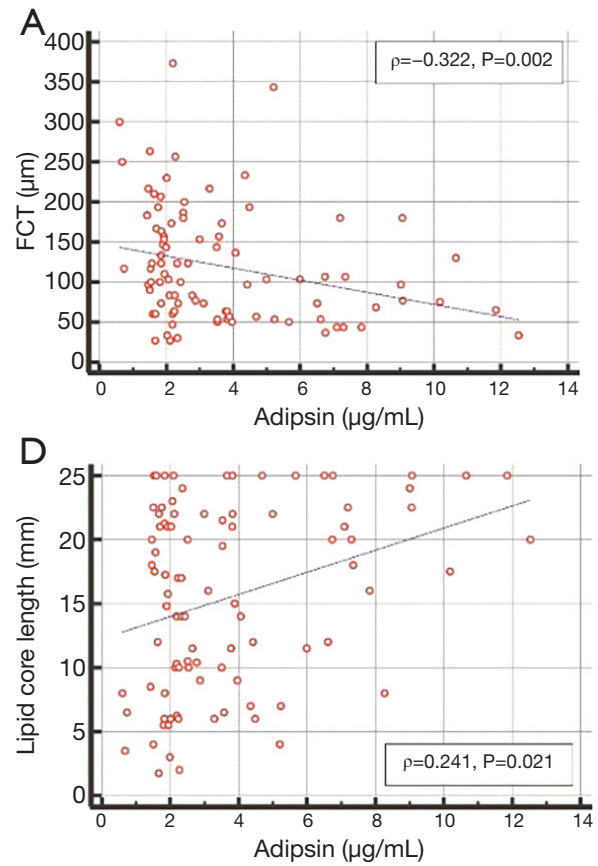

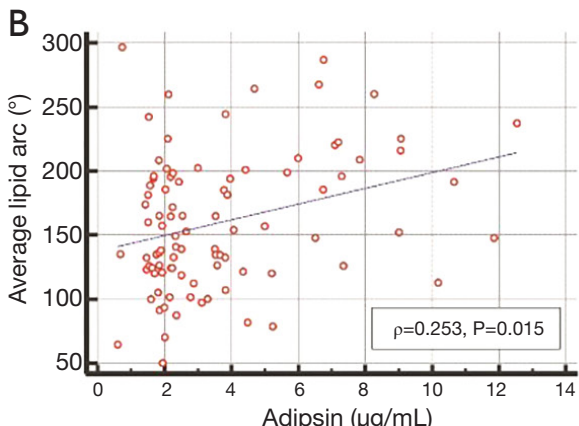

E

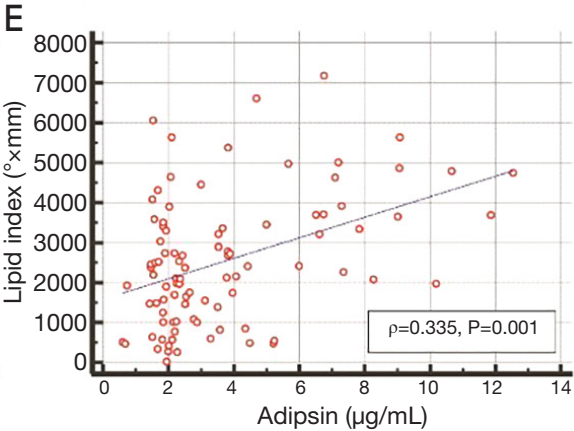

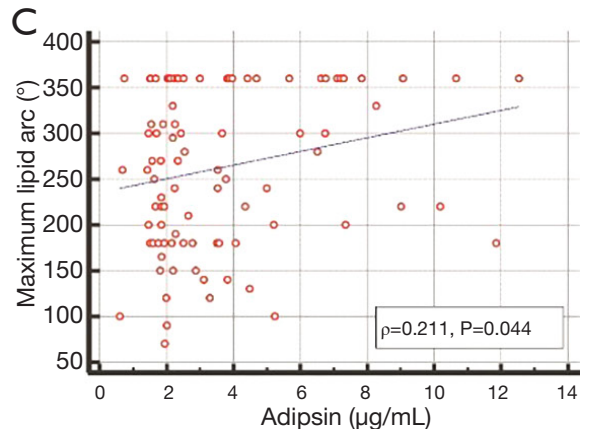

$\mathrm{F}$

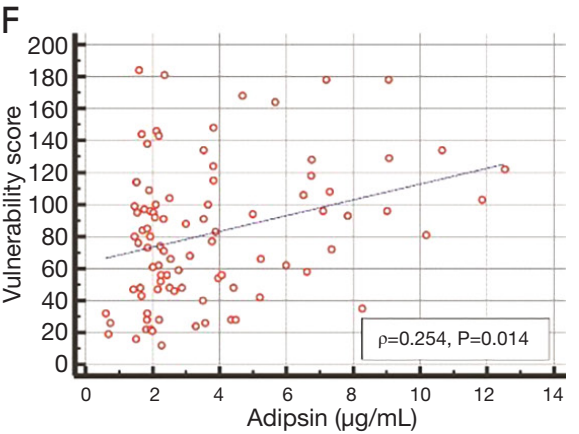

Figure 2 Correlation between serum adipsin and vulnerable plaque characteristics. Spearman correlation analysis is displayed for adipsin level and vulnerable plaque characteristics including (A) FCT, (B) average lipid arc, (C) maximum lipid arc, (D) lipid core length, (E) lipid index, and (F) vulnerability score. FCT, fibrous cap thickness.

Table 3 Univariate and multivariate analysis of possible factors associated with TCFA

\begin{tabular}{|c|c|c|c|c|}
\hline Variables & \multicolumn{2}{|c|}{ Univariate } & \multicolumn{2}{|c|}{ Multivariate } \\
\hline Age & 0.997 (0.963-1.033) & 0.888 & & \\
\hline Male & $2.097(0.851-5.164)$ & 0.107 & $2.368(0.629-8.921)$ & 0.203 \\
\hline Diabetes mellitus & $1.357(0.504-3.656)$ & 0.546 & & \\
\hline Current smoking & $1.562(0.615-3.968)$ & 0.348 & & \\
\hline TG & $1.290(0.948-1.756)$ & 0.106 & $1.006(0.559-1.813)$ & 0.983 \\
\hline $\mathrm{TC}$ & 1.463 (0.997-2.148) & 0.052 & $1.768(0.476-6.568)$ & 0.395 \\
\hline LDL-C & 1.921 (1.059-3.486) & 0.032 & $1.197(0.209-6.847)$ & 0.840 \\
\hline ACS & $5.122(1.624-16.154)$ & 0.005 & 4.058 (1.016-16.199) & 0.047 \\
\hline Visual \%DS & $1.084(1.045-1.125)$ & $<0.001$ & 1.086 (1.037-1.137) & $<0.001$ \\
\hline
\end{tabular}

\%DS, percent diameter stenosis; ACS, acute coronary syndrome; $\mathrm{Cl}$, confidence interval; HDL-C, high-density lipoprotein cholesterol; LDL-C, low-density lipoprotein cholesterol; TC, total cholesterol; TCFA, thin-cap fibroatheroma; TG, triglycerides; OR, odds ratio. 
A

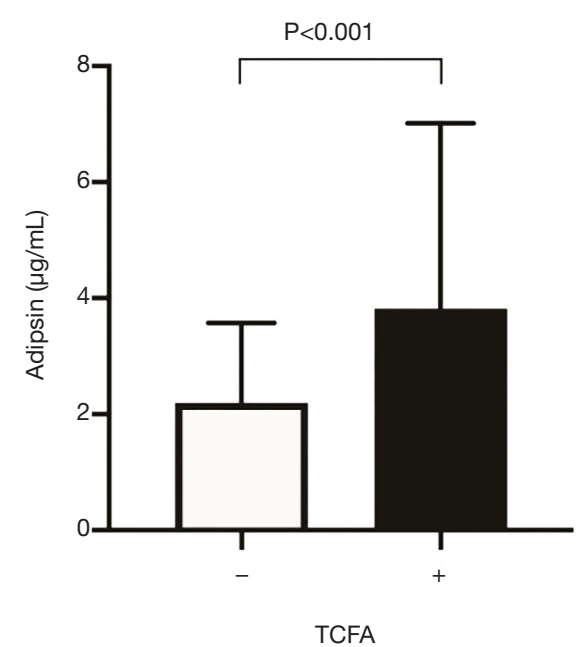

B

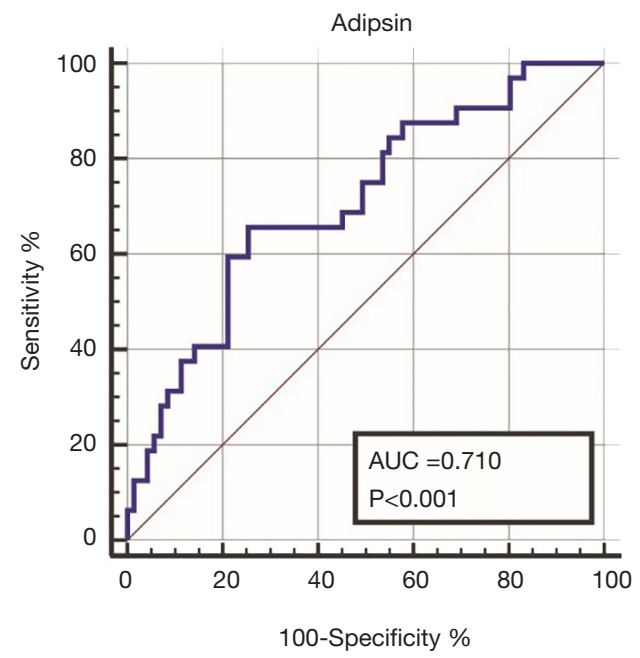

Figure 3 Association between serum adipsin and thin-cap fibroatheroma (TCFA). (A) Comparison of serum adipsin levels between the TCFA group and the non-TCFA group. The TCFA group had a significantly higher level of serum adipsin compared with the non-TCFA group (median, 3.82 vs. $2.20 \mu \mathrm{g} / \mathrm{mL}, \mathrm{P}<0.001$ ). (B) Receiver-operating characteristic (ROC) curve of adipsin for predicting the presence of TCFA. The area under the curve was 0.710 and the optimal cut-off value was $3.50 \mu \mathrm{g} / \mathrm{mL}$ (sensitivity $65.6 \%$, specificity $74.7 \%$ ).

adipsin was negatively correlated with FCT but positively correlated with average lipid arc, maximum lipid arc, lipid core length, lipid index, and vulnerability score; (II) compared with patients in the low adipsin group, those in the high adipsin group had a greater proportion of TCFAs, and serum adipsin showed moderate discriminative ability for TCFAs; and (III) adipsin was an independent factor associated with TCFAs.

The complement system is activated in atherosclerotic plaques generally through the classic, lectin, or alternative pathways (25). As a major component in the alternative pathway, adipsin cleaves complement factor B and catalyzes the formation of complement $\mathrm{C} 3$ convertase, thus triggering the hydrolysis cascade to produce complement fragments such as C3a, C3b, C5a, and C5b (9). Previous studies have indicated that adipsin may be closely related to atherosclerosis and increased cardiovascular risk $(11,26,27)$. Recently, adipsin has been demonstrated not only as a protein signature of CAD and type 2 diabetes mellitus $(28,29)$, but also as a prognostic biomarker for CAD patients (14). However, the specific role of adipsin in the pathogenesis of vulnerable plaques remains to be elucidated. In this study, we found that plaques from patients with a high circulating adipsin level exhibited a significantly higher proportion of TCFAs. It is well known that TCFA is the most significant pathological feature of vulnerable plaques $(4,30)$; therefore, a close link was established between circulating adipsin and plaque vulnerability. We further confirmed this association by observing a significant correlation between serum adipsin and several OCT-detected vulnerable characteristics. Our data showed that adipsin was a significant predictor of TCFA even after adjusting for confounding variables in the multivariate model, while ROC analysis also demonstrated its diagnostic ability to detect TCFA. Thus, adipsin may serve as an effective indicator for atherosclerosis progression and high-risk plaques to provide risk stratification and treatment guidance for patients at an early stage. Understanding its role in plaque vulnerability will be useful for developing new therapeutic targets and reducing the incidence of future adverse events.

TCFA, as a major manifestation of vulnerable plaques, is histologically characterized by a large lipid-rich necrotic core with an overlying thin fibrous cap $(\leq 65 \mu \mathrm{m})$ infiltrated by abundant inflammatory cells but rare smooth muscle cells (4). Structural stability may be determined by FCT and the size of the lipid core, both of which were found to be correlated with serum adipsin in the present study. A large lipid core may recruit more proinflammatory macrophages and increase their secretion of adipsin. Moreover, we found that serum adipsin was correlated with other OCT-detected morphological parameters, especially the vulnerability score. The score provided a quantitative 
assessment of plaque vulnerability by calculating the total number of appearances of vulnerability markers (24), which is not limited to TCFA. It has been shown that other OCT-detected plaque features, such as cholesterol crystals and macrophage accumulation, were also associated with a higher risk of coronary events (2,30). In recent years, the concept of CAD has gradually shifted from solely "vulnerable plaque" to integrated "vulnerable patient", which involves multiple factors, including atheroma burden, metabolic activity, and thrombosis formation (31). Adipsin, a lipid metabolite secreted from adipose tissue, may therefore provide additional information regarding the probability or tendency of CAD patients to develop AMI. In summary, adipsin may allow for a more comprehensive and accurate approach, not only to identify plaque vulnerability, but also to manage vulnerable patients and improve their prognosis.

The exact role of adipsin in atherosclerosis progression and plaque vulnerability, however, has not been fully elucidated. The possible mechanisms mediating this association may be as follows. First, adipsin, as a circulating protein, may reflect the level of inflammation and endothelial dysfunction, and may promote plaque progression. Atherosclerosis is a chronic, inflammatory disease; thus, inflammation plays an important role in plaque progression, vulnerability, and subsequent clinical coronary events, and is usually related to a decrease in structural stability $(32,33)$. Given its relationship with other inflammatory diseases $(34,35)$, we speculated that adipsin might also participate in the processes of atheroma formation and vascular damage, and eventually lead to plaque destabilization. Second, adipsin may function as a bridge between innate and acquired immunity in the pathogenesis of vulnerable plaques. The complement system mediates a complex immune response in a variety of inflammatory conditions, including atherosclerosis and thrombosis $(36,37)$. As a complement factor D, adipsin may play a key role in the immune and coagulation cascade due to its regulation of complement activation $(38,39)$, which in turn prompts the occurrence of AMI. Third, similar to other adipokines, adipsin may regulate endocrine function, lipid metabolism, and obesity, all of which affect the cardiovascular system and plaque stability (40-42).

Interestingly, previous investigations have identified that adipsin can maintain pancreatic beta-cell function in diabetes and that adipsin-deficient animals are prone to glucose intolerance $(43,44)$. This finding seems to be inconsistent with the current knowledge that patients with a high adipsin level are at increased risk for developing atherosclerotic cardiovascular disease $(14,26,28)$. Further studies are therefore needed to elucidate the specific effect of adipsin in CAD and how adipsin is involved in the development of vulnerable plaques. Our work revealed an association between adipsin and OCT-detected vulnerable characteristics and provided new insights into how the complement alternative pathway promotes plaque vulnerability. Activation of the alternative pathway may lead to plaque instability by influencing inflammation and endothelial function. Inhibitors of the pathway, anti-factor B and antifactor D antibodies $(45,46)$, are expected to become potential treatments for stabilizing vulnerable plaques in the future.

Several limitations of this study should be acknowledged. First, this was a single-center, prospective, observational study with a relatively small sample size. Thus, our results need to be externally validated by large, multicenter studies in the future. Second, we cannot completely rule out the potential effects of statins, antihypertensive medications, and antidiabetic medications on serum adipsin. However, we did not observe a significant difference in their baseline usage between the 2 groups. Finally, some patients had missing values of hypersensitive C-reactive protein because it was not routinely assessed in clinical practice. We did not analyze this inflammatory marker with other methods to avoid measurement bias.

\section{Conclusions}

In conclusion, serum adipsin levels tend to be associated with OCT-determined plaque vulnerability in CAD patients. Patients with a high adipsin level had a more frequent incidence of TCFAs than those who had a low adipsin level. Therefore, adipsin may be used as an effective biomarker for the early identification of vulnerable plaques and improved risk stratification of CAD patients, which in turn might translate into prognostic benefits.

\section{Acknowledgments}

We are grateful to the staff in Biobank of Zhongda Hospital, School of Medicine, Southeast University for technical assistance.

Funding: This work was supported by Jiangsu Provincial Key Research and Development Program (BE2016785), Jiangsu Provincial Key Medical Discipline (ZDXKA2016023), National Natural Science Foundation of China (81970237), and Yancheng Medical Science and Technology Development Program (YK2019006). 


\section{Footnote}

Reporting checklist: The authors have completed the STROBE checklist. Available at http://dx.doi.org/10.21037/ jtd-21-259

Data Sharing Statement: Available at http://dx.doi. org/10.21037/jtd-21-259

Conflicts of Interest: All authors have completed the ICMJE uniform disclosure form (available at http://dx.doi. org/10.21037/jtd-21-259). The authors have no conflicts of interest to declare.

Ethical Statement: The authors are accountable for all aspects of the work in ensuring that questions related to the accuracy or integrity of any part of the work are appropriately investigated and resolved. All procedures performed in this study involving human participants were in accordance with the Declaration of Helsinki (as revised in 2013). The study protocol was approved by the institutional ethics committee of Zhongda Hospital, School of Medicine, Southeast University, China, and all patients provided written informed consent.

Open Access Statement: This is an Open Access article distributed in accordance with the Creative Commons Attribution-NonCommercial-NoDerivs 4.0 International License (CC BY-NC-ND 4.0), which permits the noncommercial replication and distribution of the article with the strict proviso that no changes or edits are made and the original work is properly cited (including links to both the formal publication through the relevant DOI and the license). See: https://creativecommons.org/licenses/by-nc-nd/4.0/.

\section{References}

1. Timmis A, Townsend N, Gale C, et al. European Society of Cardiology: Cardiovascular Disease Statistics 2017. Eur Heart J 2018;39:508-79.

2. Prati F, Romagnoli E, Gatto L, et al. Relationship between coronary plaque morphology of the left anterior descending artery and 12 months clinical outcome: the CLIMA study. Eur Heart J 2020;41:383-91.

3. Stone GW, Maehara A, Lansky AJ, et al. A prospective natural-history study of coronary atherosclerosis. N Engl J Med 2011;364:226-35.

4. Virmani R, Burke AP, Farb A, et al. Pathology of the
Vulnerable Plaque. J Am Coll Cardiol 2006;47:C13-8.

5. Virmani R, Kolodgie FD, Burke AP, et al. Lessons from sudden coronary death: a comprehensive morphological classification scheme for atherosclerotic lesions. Arterioscler Thromb Vasc Biol 2000;20:1262-75.

6. Binder CJ, Chang MK, Shaw PX, et al. Innate and acquired immunity in atherogenesis. Nat Med 2002;8:1218-26.

7. Thurman JM, Holers VM. The Central Role of the Alternative Complement Pathway in Human Disease. J Immunol 2006;176:1305-10.

8. Cook KS, Min HY, Johnson D, et al. Adipsin: a circulating serine protease homolog secreted by adipose tissue and sciatic nerve. Science 1987;237:402-5.

9. White RT, Damm D, Hancock N, et al. Human adipsin is identical to complement factor $\mathrm{D}$ and is expressed at high levels in adipose tissue. J Biol Chem 1992;267:9210-3.

10. Xu Y, Ma M, Ippolito GC, et al. Complement activation in factor D-deficient mice. Proc Natl Acad Sci 2001;98:14577-82.

11. Hertle E, Arts IC, van der Kallen CJ, et al. The alternative complement pathway is longitudinally associated with adverse cardiovascular outcomes. Thromb Haemost 2016;115:446-57.

12. Stahl GL, Xu Y, Hao L, et al. Role for the Alternative Complement Pathway in Ischemia/Reperfusion Injury. Am J Pathol 2003;162:449-55.

13. Song NJ, Kim S, Jang BH, et al. Small MoleculeInduced Complement Factor D (Adipsin) Promotes Lipid Accumulation and Adipocyte Differentiation. PLoS One 2016;11:e0162228.

14. Ohtsuki T, Satoh K, Shimizu T, et al. Identification of Adipsin as a Novel Prognostic Biomarker in Patients With Coronary Artery Disease. J Am Heart Assoc 2019;8:e13716.

15. Tearney GJ, Yabushita H, Houser SL, et al. Quantification of Macrophage Content in Atherosclerotic Plaques by Optical Coherence Tomography. Circulation 2003;107:113-9.

16. Ik-Kyung J, J. TG, Briain M, Masamichi T, Fabian M, Nicusor I, et al. Jang IK, Tearney GJ, MacNeill B, et al. In Vivo Characterization of Coronary Atherosclerotic Plaque by Use of Optical Coherence Tomography. Circulation 2005;111:1551-5.

17. Tearney GJ, Regar E, Akasaka T, et al. Consensus Standards for Acquisition, Measurement, and Reporting of Intravascular Optical Coherence Tomography Studies: A Report From the International Working 
Group for Intravascular Optical Coherence Tomography Standardization and Validation. J Am Coll Cardiol 2012;59:1058-72.

18. Kato K, Yonetsu T, Kim SJ, et al. Comparison of Nonculprit Coronary Plaque Characteristics Between Patients With and Without Diabetes: A 3-Vessel Optical Coherence Tomography Study. JACC Cardiovasc Interv 2012;5:1150-8

19. Higuma T, Soeda T, Abe N, et al. A Combined Optical Coherence Tomography and Intravascular Ultrasound Study on Plaque Rupture, Plaque Erosion, and Calcified Nodule in Patients With ST-Segment Elevation Myocardial Infarction: Incidence, Morphologic Characteristics, and Outcomes After Perc. JACC Cardiovasc Interv 2015;8:1166-76.

20. Jia H, Abtahian F, Aguirre AD, et al. In Vivo Diagnosis of Plaque Erosion and Calcified Nodule in Patients With Acute Coronary Syndrome by Intravascular Optical Coherence Tomography. J Am Coll Cardiol 2013;62:1748-58.

21. Kitabata H, Tanaka A, Kubo T, et al. Relation of Microchannel Structure Identified by Optical Coherence Tomography to Plaque Vulnerability in Patients With Coronary Artery Disease. Am J Cardiol 2010;105:1673-8.

22. Kataoka Y, Puri R, Hammadah M, et al. Cholesterol Crystals Associate With Coronary Plaque Vulnerability In Vivo. J Am Coll Cardiol 2015;65:630-2.

23. Cassar A, Matsuo Y, Herrmann J, et al. Coronary atherosclerosis with vulnerable plaque and complicated lesions in transplant recipients: new insight into cardiac allograft vasculopathy by optical coherence tomography. Eur Heart J 2013;34:2610-7.

24. Park KH, Sun T, Liu Z, et al. Relationship between markers of plaque vulnerability in optical coherence tomography and atherosclerotic progression in adult patients with heart transplantation. J Heart Lung Transplant 2017;36:185-92.

25. Haskard DO, Boyle JJ, Mason JC. The role of complement in atherosclerosis. Curr Opin Lipidol 2008;19:478-82.

26. Gursoy Calan O, Calan M, Senses PY, et al. Increased adipsin is associated with carotid intima media thickness and metabolic disturbances in polycystic ovary syndrome. Clin Endocrinol (Oxf) 2016;85:910-7.

27. Ho JE, Lyass A, Courchesne P, et al. Protein Biomarkers of Cardiovascular Disease and Mortality in the Community. J Am Heart Assoc 2018;7:e008108.

28. Adela R, Reddy PNC, Ghosh TS, et al. Serum protein signature of coronary artery disease in type 2 diabetes mellitus. J Transl Med 2019;17:17.
29. Shin SK, Ha TY, McGregor RA, et al. Long-term curcumin administration protects against atherosclerosis via hepatic regulation of lipoprotein cholesterol metabolism. Mol Nutr Food Res 2011;55:1829-40.

30. Narula J, Nakano M, Virmani R, et al. Histopathologic Characteristics of Atherosclerotic Coronary Disease and Implications of the Findings for the Invasive and Noninvasive Detection of Vulnerable Plaques. J Am Coll Cardiol 2013;61:1041-51.

31. Arbab-Zadeh A, Fuster V. From Detecting the Vulnerable Plaque to Managing the Vulnerable Patient: JACC Stateof-the-Art Review. J Am Coll Cardiol 2019;74:1582-93.

32. Libby P. Inflammation in Atherosclerosis. Arterioscler Thromb Vasc Biol 2012;32:2045-51.

33. Gough PJ, Gomez IG, Wille PT, et al. Macrophage expression of active MMP-9 induces acute plaque disruption in apoE-deficient mice. J Clin Invest 2006;116:59-69.

34. Li Y, Zou W, Brestoff JR, et al. Fat-Produced Adipsin Regulates Inflammatory Arthritis. Cell Rep 2019;27:28092816.e3.

35. Schmid A, Hochberg A, Berghoff M, et al. Quantification and regulation of adipsin in human cerebrospinal fluid (CSF). Clin Endocrinol (Oxf) 2016;84:194-202.

36. Speidl WS, Kastl SP, Huber K, et al. Complement in atherosclerosis: friend or foe? J Thromb Haemost 2011;9:428-40.

37. Rittirsch D, Flierl MA, Ward PA. Harmful molecular mechanisms in sepsis. Nat Rev Immunol 2008;8:776-87.

38. Wu X, Hutson I, Akk AM, et al. Contribution of AdiposeDerived Factor D/Adipsin to Complement Alternative Pathway Activation: Lessons from Lipodystrophy. J Immunol 2018;200:2786-97.

39. Dunkelberger JR, Song WC. Complement and its role in innate and adaptive immune responses. Cell Res 2010;20:34-50.

40. Gasbarrino K, Zheng H, Hafiane A, et al. Decreased Adiponectin-Mediated Signaling Through the AdipoR2 Pathway Is Associated With Carotid Plaque Instability. Stroke 2017;48:915-24.

41. Lau WB, Ohashi K, Wang Y, et al. Role of Adipokines in Cardiovascular Disease. Circ J 2017;81:920-8.

42. Gasbarrino K, Mantzoros C, Gorgui J, et al. Circulating Chemerin Is Associated With Carotid Plaque Instability, Whereas Resistin Is Related to Cerebrovascular Symptomatology. Arterioscler Thromb Vasc Biol 2016;36:1670-8.

43. Gómez-Banoy N, Guseh JS, Li G, et al. Adipsin preserves 
beta cells in diabetic mice and associates with protection

from type 2 diabetes in humans. Nat Med 2019;25:1739-47.

44. Lo JC, Ljubicic S, Leibiger B, et al. Adipsin Is an Adipokine that Improves $\beta$ Cell Function in Diabetes. Cell 2014;158:41-53.

45. Fung $M$, Loubser PG, Ündar A, et al. Inhibition of complement, neutrophil, and platelet activation by an anti-factor D monoclonal antibody in simulated cardiopulmonary bypass circuits. J Thorac Cardiovasc

Cite this article as: Sun R, Qiao Y, Yan G, Wang D, Zuo W, Ji Z, Zhang X, Yao Y, Ma G, Tang C. Association between serum adipsin and plaque vulnerability determined by optical coherence tomography in patients with coronary artery disease. J Thorac Dis 2021;13(4):2414-2425. doi: 10.21037/jtd-21-259
Surg 2001;122:113-22.

46. Leinhase I, Rozanski M, Harhausen D, et al. Inhibition of the alternative complement activation pathway in traumatic brain injury by a monoclonal anti-factor B antibody: a randomized placebo-controlled study in mice. J Neuroinflammation 2007;4:13.

(English Language Editor: C. Betlazar-Maseh) 